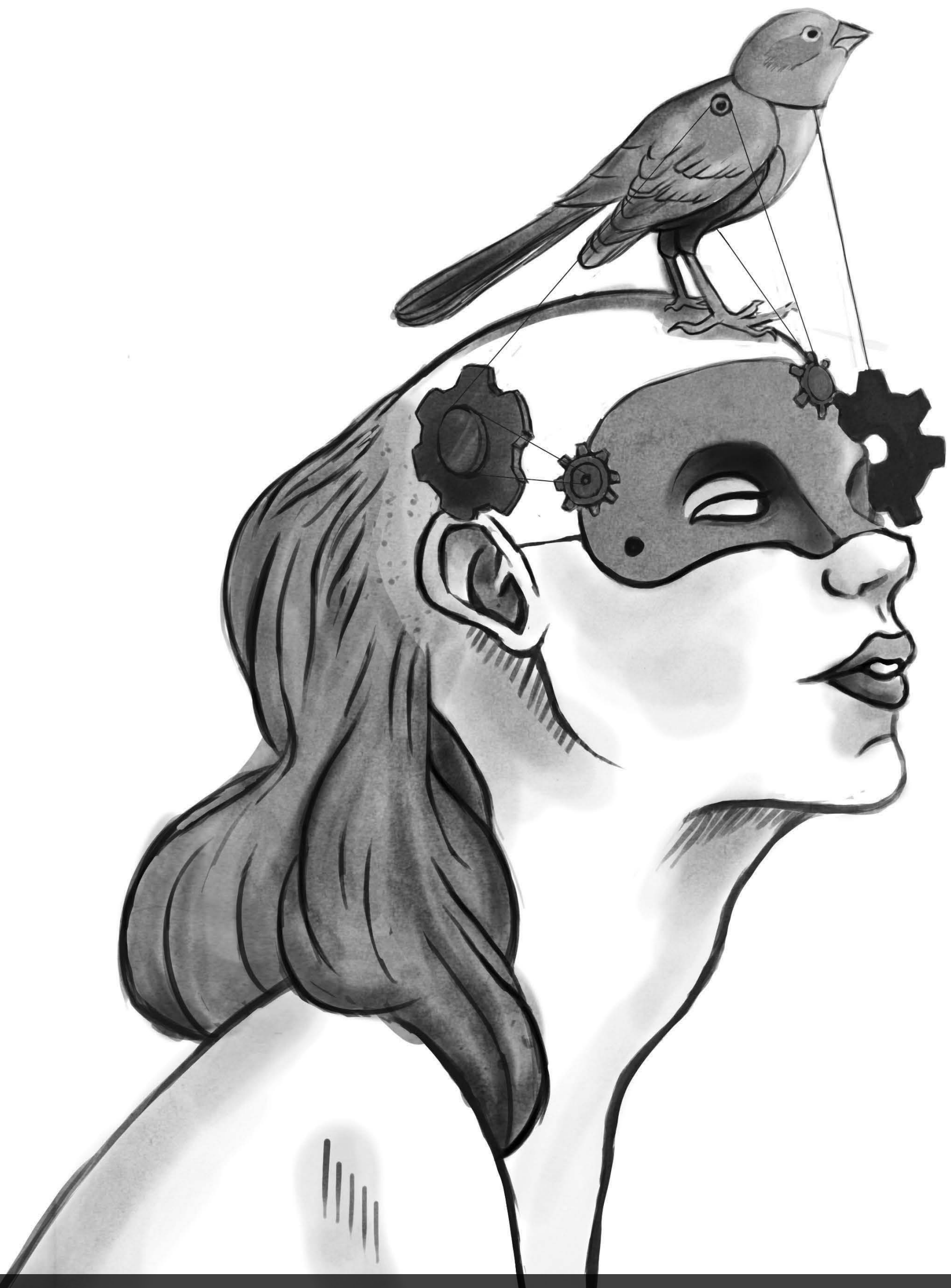

Hacia una comprensión de la mente y el comportamiento del emprendedor 


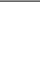




\title{
Hacia una comprensión de la mente y el comportamiento del emprendedor ${ }^{1}$
}

Fecha recepción: 3 de noviembre de 2010

Fecha de aprobación: 18 de enero de 2011

\author{
Álvaro Rolando Bonilla Ballesteros \\ Politécnico Grancolombiano \\ (Colombia) \\ abonilla@poli.edu.co \\ Psicólogo. Universidad Javeriana. Director del \\ Departamento de Humanidades del Politécnico \\ Grancolombiano.
}

\section{Resumen}

Este artículo se pregunta por los procesos psicológicos involucrados en el emprendimiento empresarial, y cómo es posible articular un proyecto educativo que dé cuenta de los elementos necesarios para elaborar un proyecto de vida basado en proyectos emprendedores. Se detallan los procesos de percepción, autoeficacia, intención, motivación, emoción y atribución y se retoman sus efectos en el tránsito desde la intención de ser emprendedor hasta el comportamiento emprendedor. La discusión propone tendencias de investigación y temáticas clave para desarrollar pedagogías y metodologías de una cátedra de emprendimiento.

\section{Palabras clave}

Psicología, comportamiento, emprendimiento, percepción, motivación, emoción, autoeficacia, intención, atribución, educación.

\section{Abstract}

This article examines the question of the psychological processes involved in entrepreneurship and the possibility of articulating an educational project that provides for the necessary elements to develop a life plan based on entrepreneurship. Processes of perception, self-efficacy, intention, motivation, emotion, and attribution are detailed, and their effects on the transition from the intention of being an entrepreneur to entrepreneurial behavior are taken up again. The discussion proposes research trends and key issues to develop pedagogies and methodologies of a chair in entrepreneurship.

\section{Keywords}

Psychology, Behavior, Entrepreneurship, Perception, Motivation, Emotion, Self-Efficacy, Intention, Attribution, Education. 


\section{Introducción}

El interés de este abordaje del emprendimiento, parte de una inquietud personal del autor, que podría expresarse así: ¿Cuáles son las características, en términos de comportamiento, percepción, motivación y emoción, de los emprendedores que se deciden por una aventura empresarial y de negocios? En últimas, ¿qué caracteriza a las personas, en términos de conducta y mente, cuando inician un nuevo proyecto empresarial? La respuesta a esta inquietud es, de por sí, bastante ambiciosa, puesto que puede darnos ideas sobre procesos como la creación de ideas, la innovación, el liderazgo y la toma de decisiones.

Las respuestas arrojadas pueden contribuir a que la psicologia aborde, de frente y sin rodeos, un proceso en el cual ha tardado en hacerse notar -más no en llegar, como se mostrará en la revisión de autores-, para que sea parte de un trabajo necesariamente interdisciplinario junto con administradores de empresas, economistas y sociólogos, los cuales intentan aproximarse a la génesis del emprendimiento en la mente humana, al origen de la puesta en acción de una idea y a lo que en últimas lleva al éxito y al fracaso de una iniciativa emprendedora.

Esta investigación se enmarca dentro del contexto de la ley 1014 de 2006, en la cual se promueve la cultura del emprendimiento. En esta ley se define la cultura como "conjunto de valores, creencias, ideologias, hábitos, costumbres y normas, que comparten los individuos en la organización y que surgen de la interacción social, los cuales generan patrones de comportamiento colectivos que establece una identidad entre sus miembros y los identifica de otra organización". La intuición que siguió esta investigación fue la estudiar el emprendimiento a partir de los procesos psicológicos del emprendedor o el aspirante a emprendedor, teniendo en mente que la orientación de los procesos de acompañamiento y fomento de la cultura emprendedora no han acudido a los conocimientos que desde la psicologia se han logrado del estudio del proceso emprendedor y sus iniciativas empresariales.

En el artículo segundo, la Ley define uno de sus objetivos principales: "crear un vínculo del sistema educativo y sistema productivo nacional mediante la formación en competencias básicas, laborales, ciudadanas y empresariales a través de una cátedra transversal de emprendimiento" dentro de este marco, es posible identificar la importancia que reviste el desarrollo de competencias dentro del proceso formativo del emprendedor. La psicología ha identificado en los procesos básicos de percepción, motivación, emoción, autoeficacia y atribución cuyo conocimiento es fundamental con miras a orientar al emprende-dor desde el momento en que siente el impulso de aventurarse en un emprendimiento hasta el momento en que lleva su iniciativa a cabo, convirtiéndose en un emprendedor efectivo.

Es importante el conocimiento psicológico con el propósito de no sólo preparar al futuro emprendedor en el diseño de un plan de negocio, en la consideración de las variables económicas, humanas y técnicas de su proyecto, sino que es fundamental, de acuerdo con la psicologia, prepararlo para distorsiones perceptivas, para entender cómo las emociones facilitan o entorpecen sus decisiones, para desarrollar una comprensión sobre cómo los procesos de motivaciones operan sobre la toma de riesgos, y cómo las atribuciones y lugares de control percibido pueden interferir en procesos de diagnóstico.

El abordaje de la cultura del emprendimiento, desde la óptica de la psicología, es un campo que aún necesita desarrollos experimentales que puedan dar cuenta de lo que pasa por la mente de un emprendedor en momentos criticos de su proceso. Lo anterior puede darnos pistas sobre cuáles son las rutas que conducen a las mejores decisiones y cuáles entorpecen los criterios de búsqueda de información y que en últimas, conducen a que el emprendedor se lance en iniciativas que al final conducirán a resultados negativos, pérdidas económicas o incluso quiebras.

\section{Marco teórico}

Durante la década de los noventa, el campo de la investigación sobre el emprendimiento no habia podido demostrar algún rasgo único que diferenciara la personalidad del emprendedor (Brockhaus \& Horwitz, 1986). Muchos teóricos fracasaron en su búsqueda del santo grial de lo que hacía diferente a un emprendedor, su búsqueda estaba sesgada a ver al emprendedor como una especie fuera de serie y a la cual correspondian procesos únicos de pensamiento y comportamiento. La investigación actual ha trascendido esa tendencia y 
actualmente intenta concentrarse en conocer cuáles son los procesos críticos que enfrenta un emprendedor en su camino, asumiendo que un emprendedor exitoso tiene rasgos similares a cualquier otro profesional exitoso o lider que ha decidido ser un empleado (Carsrud et al., 1989, Carter et al., 2004). El camino tomado por la literatura revisada se enfoca, más bien, en buscar y adoptar modelos interpretativos y teorias a partir de la psicología y otras ciencias como por ejemplo el mercadeo, que permitan ser usadas para una mejor comprensión de las cogniciones, motivaciones y comportamientos del emprendedor.

El primer proceso que se revisará será el de percepción. Este proceso en los emprendedores resulta fundamental, en la medida en que puede explicar por qué los emprendedores dan un paso adelante al iniciar una aventura empresarial mientras que otros retroceden y se deciden por la estabilidad del negocio que les ofrece una organización ya constituida.

Desde el momento en que un individuo se convierte en un emprendedor naciente que activamente planea el inicio de su propio negocio, y pasa a una fase exploratoria, comienza una búsqueda por la información relevante que contribuya a la reducción de riesgos potenciales en el marco de la investigación por la viabilidad de su proyecto. El emprendedor naciente busca datos acerca de la disponibilidad y la accesibilidad de los recursos requeridos para lanzarse a su nueva idea de negocio. En algún punto, este emprendedor forma la creencia de que ha reunido suficiente información y decide explotar su oportunidad, pasando de ser un aspirante a emprendedor a un emprendedor práctico. El proceso de emprendimiento tiene lugar en un ambiente de alta incertidumbre, de tal manera que cuando se introducen nuevos productos, nuevos servicios o nuevos modelos de negocios no es posible predecir con precisión los resultados de las decisiones que se toman (Morris et al., 2006).

En cada fase del proceso de emprendimiento, las percepciones juegan un papel clave en conducir al individuo a convertirse en un emprendedor práctico. Al comienzo del proceso emprendedor, los individuos forman la intención de llegar a ser emprendedores y entrar en la fase de exploración (McMullen \& Shepherd, 2006; Choi et al., 2008). La formación de la intención emprendedora puede anteceder, o seguir, el descubrimiento de una oportunidad especifica para ser explotada. Para algunos, la formación de la intención general de convertirse en emprendedor dispara la búsqueda de una oportunidad deseable, mientras que para otros el descubrimiento de una oportunidad deseable dispara la formación de intenciones de emprendimiento. Bhave (1994) llama al primer caso "reconocimiento de oportunidad internamente estimulada" y al segundo caso "reconocimiento de oportunidad externamente estimulada".

La percepción puede entenderse como la forma en que miramos la realidad, los lentes que traemos puestos y que influyen en la forma en que apreciamos las variables a las que decidimos prestar atención. Los emprendedores pueden tener unos lentes óptimos o unos lentes que pueden hacer que la realidad aparezca distorsionada. El efecto de unos lentes adecuados, corresponde a una gran autoeficacia para tareas emprendedoras que resultan de un conocimiento óptimo capitalizado a nivel individual y de un capital social amplio que permiten al emprendedor ver demandas $y$ necesidades futuras de nuevos productos, servicios y procesos de negocio y predecir la evolución de una nueva tecnología que sirva a las preferencias del consumidor y a las necesidades del mercado (Gimeno et al., 1997).

Krueger (1993), Krueger y Brazeal (1994) y Krueger y Carsrud (1993), argumentan que los dos principales factores que subrayan la formación de las intenciones de emprendimiento son la viabilidad percibida y la conveniencia percibida de la oportunidad emprendedora. McMullen y Shepherd (2006) consideran que conocimiento y motivación son los principales motores de la decisión de explotar la oportunidad.

Existen problemas de percepción que requieren la atención de educadores y formadores del talento emprendedor, así como de accionistas e inversionistas. La excesiva confianza en sus habilidades que caracteriza a algunos emprendedores nacientes (Palich \& Bagby, 1995), causa un optimismo desmesurado (Simon, et al., 2000), que estima como más grandes las oportunidades de lo que en realidad son y sobreestima las habilidades propias para resolver problemas, para lograr beneficios financieros, para cumplir metas y juzgar preferencias de los consumidores, por mencionar algunas. Simon et al. (2000) distinguen entre la excesiva confianza, definida como la pérdida de conocimiento sobre los limites del conocimiento propio (Russo \& Shoemaker, 1998) y la ilusión de control, que consiste en la 
sobreestimación de la habilidad propia para controlar los eventos futuros en situaciones inciertas (Larger, 1975). Boyd \& Vozikis (1994), argumentan que la ilusión de control afecta positivamente la formación de la intención emprendedora. Dentro de la crucial fase de exploración, estas distorsiones pueden inhibir la precisión del emprendedor acerca de necesidades del mercado y estimaciones de costos, haciendo que el emprendedor inicie su aventura empresarial cuando otros probablemente habrian abandonado.

Otra forma de distorsión es la tendencia de algunos emprendedores a apasionarse por el proceso de emprendimiento y por los logros y reconocimientos que están asociados con el convertirse y ser un emprendedor, elementos que conducen a que el individuo siga adelante, ignorando información importante, impulsado por los beneficios intrínsecos asociados a la actividad emprendedora. Los emprendedores pueden percibir más intensamente los beneficios emocionales asociados con una oportunidad de emprendimiento. Aunque comúnmente pensamos en ganancias y crecimiento como los principales objetivos de los emprendedores, ellos persiguen la actividad también por beneficios no monetarios tales como los beneficios psicológicos asociados con convertirse en emprendedores. Algunos de los beneficios más comúnmente citados son "ser mi propio jefe" (Barringer e Ireland, 2006, p. 6-7; Shane, 2003, p. 106). Varios estudios han demostrado que la preferencia por la independencia es significativa y positivamente asociada a la formación de intenciones emprendedoras (Douglas \& Shepherd, 2002) y distingue de forma significativa a los emprendedores de los no emprendedores (Shane, 2003).

Por último, puede darse entre los emprendedores una distorsión basada en la tendencia a ver las oportunidades más grandes de lo que son, más cercanas en el tiempo y menos riesgosas de lo que en realidad son. Es importante anotar que el exceso de confianza no es igual a la sobreestimación de los resultados o la minimización de los riesgos (Sitkin \& Pablo, 1992). El exceso de confianza se relaciona con una autoeficacia que excede la capacidad actual del individuo para completar una tarea de forma exitosa. La sobreestimación, por su parte, tiene que ver con el fallo del individuo para estimar de forma correcta el tamaño y la complejidad de una situación dentro de un contexto de emprendimiento.
Ahora bien, dentro del emprendimiento, un proceso importante es el de las intenciones del individuo de implementar una conducta emprendedora. Krueger et al. (2000) desarrollaron un modelo de las intenciones del emprendedor, que les permitió concluir que los eventos emprendedores son el resultado de factores socioculturales y situaciones que interactúan. Cada evento de emprendimiento ocurre como resultado de un proceso dinámico que provee el impulso situacional que tiene en los individuos cuyas percepciones y valores están determinados por su herencia social y cultural y su previa experiencia. El modelo de las intenciones del emprendedor asume que la viabilidad percibida y la conveniencia percibida predicen la intención de llegar a ser un emprendedor. Las normas sociales percibidas y la autoeficacia percibida operan como antecedentes de la viabilidad y la conveniencia percibida (Krueger \& Brazeal, 1994).

El estudio de las intenciones del emprendedor puede ser entendido sólo como un marco teórico donde la motivación, metas y oportunidades de evaluación están incluidas. La estructura del proceso de formación de intención del emprendedor posee el poder de provocar el comportamiento emprendedor (con la intermediación de las metas emprendedoras) y, como tal, es útil para acercarnos a la conducta del emprendedor. La existencia de diferentes clases de metas, en este caso metas de orden superior y metas emprendedoras, refleja la jerarquía de metas introducida por Bagozzi y Dholakia (1999). Las metas emprendedoras pueden ser metas focales o metas subordinadas. Las intenciones de emprendimiento son principalmente resultado de metas de orden superior, la viabilidad percibida, la conveniencia percibida y la evaluación de la oportunidad. En el contexto del modelo, estas variables constituyen un círculo alrededor de la intención emprendedora. Las variables dentro del círculo se afectan de forma reciproca. Los resultados de Elfving (2008) indican que las metas de orden superior afectan tanto la percepción de la conveniencia percibida como la percepción de la viabilidad percibida. Si la meta principal es ganar independencia, la viabilidad y conveniencia percibida serán evaluadas en relación con cuánta independencia pueden proveer.

Si un individuo percibe el emprendimiento como viable, conveniente y deseable, considera al emprendimiento como una actividad en linea con sus metas generales en la vida $\mathrm{y}$ ve la oportunidad de desempeñarse como 
emprendedor, entonces tiene la tendencia de formar una intención emprendedora. La motivación aparece como una variable importante, en la medida en que determina qué clase de metas de orden superior desarrolla una persona en su vida. Las metas de orden superior están construidas en relación con lo que es percibido como motivador. En este punto la autoeficacia es importante, porque si la motivación determina lo que una persona desea ser, la autoeficacia determina lo que el individuo piensa que puede hacer. La autoeficacia impacta tanto a las metas de orden superior como a las metas emprendedoras. Sin embargo, es importante recordar que la autoeficacia es específica a un contexto y contenido (Bandura, 1986, 1989), y ambas clases de metas tienden a verse afectadas por diferentes clases de autoeficacia; ésta impacta a la motivación a través del compromiso, del cual también habla Bandura (1989) en su investigación. Una alta autoeficacia mejora el compromiso y hace que la persona se encuentre más motivada para continuar.

Es momento de revisar un núcleo importante del comportamiento emprendedor: la motivación. De acuerdo al Monitor de Emprendimiento Global (GEM, por sus siglas en inglés), una motivación orientada a la sobrevivencia puede ser vista como la "necesidad emprendedora" (Reynolds et al., 2002). El ser humano se encuentra involucrado en un mundo moderno, donde se obtiene lo necesario para sobrevivir trabajando con el fin de obtener medios monetarios para adquirir lo que se necesita y desea. La mayoria de personas realizan esto trabajando como empleados, para corporaciones $\mathrm{u}$ otro tipo de organizaciones. Sin embargo, no todos desean asumir el rol de empleado trabajando para otra persona dentro de una empresa. Algunos deciden tomar su propio camino a través del mundo de los negocios como emprendedores.

El sociólogo Homans (1961) propuso los principios motivaciones del hedonismo y la teoría del "hombre económico" que aún tiene relevancia al estudiar el emprendimiento. E1 énfasis utilitarista en el papel de la "recompensa", "reducción de la pulsión", "placer", "refuerzo" o "satisfactores", tal como ha sido propuesta en las teorías de la motivación psicológicas, puede brindar información importante a la hora de estudiar los retos que se propone el individuo emprendedor (Deutsh \& Krauss, 1965).
Tradicionalmente la motivación ha sido estudiada para responder a tres clases de preguntas: (i) ¿Qué activa a una persona? (ii) ¿Qué hace que emprendan uno $u$ otro reto? (iii) ¿Por qué las personas responden de formas diferentes a los mismos estímulos? Estas preguntas conducen a tres importantes aspectos de la motivación: la activación, la selección-dirección y la respuesta dispuesta (Perwin, 2003). Las teorias actuales de la motivación se pueden dividir en teorias de la pulsión y teorias de la recompensa. Las teorias de la pulsión sugieren que existe un estimulo interno que orienta a la persona a buscar la reducción de la tensión, siendo la necesidad de reducir la tensión una motivación (Freud, 1924; Murray, 1938; Festinger, 1957). Las teorias de la recompensa, por otro lado, enfatizan la fuerza motivacional que tienen los incentivos (Carsrud et al., 1989).

Fisher (1930) destaca que existen dos escuelas de teorías de la motivación, una basada en la economía y otra basada en la psicologia, las cuales han estado en conflicto por décadas. Recientemente Steel \& König (2006) y Wilson (1998), invocaron la necesidad de una conciliación, la cual es descrita por los autores como la necesidad de vincular los hechos y teorias de ambas disciplinas con el fin de crear un marco teórico común entre las dos escuelas. Es posible ver esta falta de conciliación entre las dos disciplinas en el campo específico del estudio del emprendimiento.

La complejidad de las motivaciones puede verse en dos hechos: la disonancia cognitiva y la evitación del riesgo, las cuales son fuertes motivaciones de los seres humanos. La investigación de la disonancia cognitiva y la necesidad de evitar el error (Cohen \& Zimbardo, 1969) puede ser empleada para explicar por qué los emprendedores a menudo hacen lo que sea para evitar fallar en sus emprendimientos. Por ejemplo, las personas con una elevada motivación hacia la realización mostrarán una mayor disonancia cognitiva entre más fuerte sea la probabilidad de error (Cohen \& Zimbardo, 1969). Para reducir la disonancia, el emprendedor tendría dos alternativas: o disminuir su motivación de realización o su motivación de evitar el error. Existe un promisorio terreno de investigación en profundizar cuáles son las estrategias de reducción de la disonancia que poseen los emprendedores.

Aunque la motivación puede existir de muchas maneras, en últimas proviene de dos fuentes: 
desde el interior del yo y desde el ambiente externo. La motivación puede venir internamente, desde el impulso mocional que alguien siente al iniciar un negocio, o externamente desde la admiración de un gremio o un grupo de individuos o el dinero recibido por el emprendimiento; de esta manera la motivación puede ser intrínseca o extrinseca. La motivación intrínseca se refiere a un interés personal en la tarea, como lo es la motivación del logro (Carsrud et al., 1989), y la motivación extrínseca se refiere a una recompensa externa que sigue a cierto comportamiento (Perwin, 2003; Nuttin, 1984). Ambos tipos de motivación no son mutuamente excluyentes, uno puede ser motivado por ambas al momento de realizar una tarea (Nuttin, 1984; Elfving, 2008).

La motivación del emprendimiento está vinculada tanto a factores internos como a factores externos (Elfving, 2008). Internamente, los emprendedores pueden estar motivados por el hecho de ser sus propios jefes y obtener riqueza financiera. Mientras la mayoria de investigaciones apuntan a que la motivación para el emprendimiento está orientada por las recompensas externas, tales como el dinero, poder o estatus (un punto de vista económico de la motivación humana), es necesario empezar a discernir cuáles son las motivaciones que se encuentran en el individuo y que hacen que surja la intención emprendedora. La definición de motivación intrínseca apunta a que es necesaria para la automotivación y la integración de la personalidad que incluiria la necesidad de ser competente, de conectarse y de ser autónomo (Deci, 1975). Esta definición sería útil para entender por qué algunos emprendedores inician emprendimientos sociales incluso cuando no existe una recompensa aparentemente más que la satisfacción personal.

Los emprendedores a menudo han sido descritos como inmersos totalmente en sus emprendimientos e incluso comprometidos al punto de la obsesión con sus iniciativas. Koch (1956) apunta que quienes se involucran en tareas por motivación intrínseca estarian más organizados y energizados. Esto podría explicar por qué el panel de estudios del emprendimiento citado por Reynolds et al. (2002) encuentra que quienes no pueden iniciar exitosamente algún emprendimiento dicen que lo intentarian con un nuevo emprendimiento. El haber abandonado una iniciativa y aún querer intentarlo de nuevo es un indicador de motivación intrínseca.
Otra forma de abordar las motivaciones intrinsecas y extrinsecas, es mirar la satis-facción en la vida y el trabajo: estas son fuerzas motivacionales intensas para la mayoría de los individuos. El malestar en un trabajo actual puede hacer que un empleado piense en la opción de convertirse en un emprendedor. Recortes de personal, condiciones económicas particulares y otras fuerzas fuera del control personal pueden forzar la motivación para valerse económicamente por una iniciativa propia (Elfving, 2008). Aunque también es cierto el hecho de que las personas dejan empleos seguros y satisfactorios para convertirse en emprendedores.

Las metas pueden ser vistas como representaciones mentales o esquemas de lo que se espera del futuro y que impulsan a los individuos a no desanimarse (Perwin, 2003). Las metas son unidades centrales en la teoria de Bandura. De acuerdo a este autor, la autoeficacia es en parte responsable de determinar lo que las personas intentan lograr y cuáles metas establecen (Bandura, 1989). Las metas activan a las personas y sirven como un importante vínculo entre la intención y la acción (Perwin, 2003; Nuttin, 1984). Las metas juegan un rol importante en la predicción del comportamiento humano.

De acuerdo a Locke y Latham (2002), las metas tienen una función directiva, ayudan a enfocar la atención y esfuerzos hacia actividades importantes para la consecución de la meta e ignorar las actividades que son irrelevantes. Las metas sirven como energizadores. Entre más alta es la meta, más grandes son los esfuerzos que los individuos hacen para lograrlas (Bandura, 1989). Las metas afectan la persistencia: entre más alta es la meta, más grande es la voluntad para trabajar por ella. Por último, las metas pueden dar lugar a la emergencia de estrategias para lograrlas. La relación entre metas y desempeño es más fuerte en personas altamente comprometidas. La existencia de la retroalimentación es otro factor importante en la teoria de las metas. Las personas necesitan ser capaces de verificar en qué lugar se encuentran en relación con su meta para determinar qué ajustes es necesario realizar en su comportamiento para lograrla (Lent et al., 1994).

Bagozzi \& Dholakia (1999) sugieren que las metas pueden ser divididas en tres niveles: metas focales, metas subordinadas de nivel inferior, y metas de orden superior. La meta 
focal se ubica en el centro de la jerarquía y responde a la pregunta ¿Qué es lo que busco? La meta subordinada de nivel inferior responde a la pregunta ¿Cómo puedo lograr lo que busco? Y la meta de orden superior responde a la pregunta ¿Por qué quiero lograr lo que me he propuesto buscar?

El trabajo de Gollwitzer \& Brandstätter (1997) ilustra la diferencia entre intenciones, motivación y metas, y además presenta las ideas de intenciones de implementación y búsqueda de metas. La búsqueda de metas es un continuo que incluye cuatro fases de acción. La primera fase es la fase predecisional, que es un despertar de deseos. En la segunda fase, la preaccional se inicia el comportamiento orientado. En la tercera fase, de acción, las acciones orientadas a metas están enfocadas a un desenlace exitoso. Finalmente, en la cuarta fase, post acción, los resultados son evaluados comparando lo que se ha obtenido con lo que originalmente se deseaba.

Las cuatro fases de acción están conectadas por ciertos puntos de transición. Gollwitzer y Brandstätter (1997) etiquetan el primer punto de transición como intención de meta, por ejemplo cuando un individuo afirma "Yo intento llegar a ser un emprendedor". Sin embargo, una intención no es suficiente para llevar a la acción, puesto que muchos impedimentos pueden presentarse en el camino. De otra parte, un individuo puede escoger entre diferentes caminos para alcanzar una meta con el fin de evitar al riesgo de fracasar en una oportunidad específica. Una intención de implementación puede funcionar como un mediador y llevar la búsqueda de la meta un paso adelante. Sirve para traducir el estado de la meta desde un nivel de mayor abstracción hacia un nivel inferior y para vincular un cierto comportamiento orientado a metas a un contexto específico. Una intención de implementación podría ser por ejemplo expresada con la frase "Pretendo iniciar mi propio negocio cuando haya finalizado mis estudios". Una intención de implementación se refleja en un compromiso de desempeñar un comportamiento específico orientado a una meta una vez que ha ocurrido una situación crítica. Las personas que han desarrollado una intención de implementación deberían poseer las estructuras cognitivas necesarias para reconocer las oportunidades cuando éstas emergen. Gollwitzer \& Brandstätter (1997) concluyen que una meta tiene una mayor tendencia de lograrse cuando existe una intención de implementación.
Bagozzi et al. (2003) han añadido la intención de implementación a su modelo original (Bagozzi \& Warshaw, 1992). El modelo sugiere que las decisiones comportamentales son realizadas a dos niveles: primero al nivel de metas (o intenciones de metas), y segundo al nivel de acción necesaria para lograr la meta (intención de implementación). El rol mediador de los constructos motivacionales (meta y deseos de implementación), constructos emocionales (emociones anticipatorias tanto positivas y negativas) y constructos actitudinales (actitudes, normas sociales, viabilidad, confianza, control percibido) son tomadas en cuenta en el modelo.

Si tomamos la discusión de motivaciones intrínsecas y extrínsecas y la vinculamos en la discusión de metas y cogniciones, es posible crear una descripción de las características que tienen los diferentes tipos de emprendedores (Elfving, 2008), y han sido compendidas por el autor en el siguiente modelo: 


\section{Emprendedores extrínsecos}

\section{Emprendedores extrínsecos e intrínsecos}

\section{Emprendedores intrínsecos}

Motivación - Motivación extrínseca.

- Enfocados alrededor de una actividad emprendedora específica.

- Creatividad y resultado como motivadores.
- Una mezcla de motivaciones intrínsecas y extrínsecas.

- Enfocados alrededor de la iniciativa.- La independencia como motivador.
- Motivación intrínseca.

- Centrados alrededor del emprendedor.

- Redes e influencia como motivadores.

\section{Cognición}

- Alta autoeficacia que deja paso al desempeño del negocio.

- No está enfocado en el pensamiento visionario.

- Reconocen oportunidades.

- No saben cómo buscar información.

- La intuición influencia la toma de decisiones.
- Alta autoeficacia orientada al negocio y a sus habilidades.

- Enfocado al pensamiento analítico.

- Descubren oportunidades.

- Usan la información.

- El análisis influencia la toma de decisiones.
- Alta autoeficacia orientada al emprendimiento.

- No son enfocados aunque tienen pensamiento innovador.

- Creadores de oportunidad.

- No tienen tiempo para la búsqueda de información.

- Toman decisiones con base en heurísticos.
Metas una meta subordinada.

- Las metas emprendedoras son menores, se orientan a la sobrevivencia.

- Quieren tanto ganancias como sobrevivir.
- El emprendimiento es una meta focal.

- Tienen metas estratégicas y racionales.

- Enfocado a las ganancias.
- El emprendimiento es una meta focal.

- Las metas pueden ser elevadas y algo abstractas.

- Ser rentable es visto como una meta interesante. 
Las emociones y las cogniciones interactúan e influyen en el proceso del emprendimiento y en el proceso de toma de decisión. Las emociones en el proceso de emprendimiento no han sido examinadas muy detenidamente (Cardon et al., 2005; Goss 2005, 2007, Shepherd. 2004), sin embargo en la literatura del emprendimiento es posible rastrear la conexión con el proceso de la toma de decisiones (Baron, 2000a; Goss, 2007). El emprendedor se enfrenta a tareas específicas en contextos de cambios constantes e impredecibles, y al no poder tomar decisiones basado en guiones previamente construidos a menudo tiene que confiar en su "intuición" o en sus "sentimientos viscerales", los cuales bajo ciertas circunstancias son especialmente fuertes (Baron, 2008). Las emociones influencian el proceso de toma de decisiones y los juicios, y considerando la tesis de que los individuos pueden controlar o incluso suprimir sus emociones positivas o negativas, de acuerdo a ciertos estudios (Shiv et al., 2005, Spencer, 2005) se ha probado que los individuos que toman decisiones de forma independiente a sus emociones son más exitosos y eficientes en su proceso de toma de decisiones. En relación a las emociones, Baron (1998, 2000b, 2008) postula que los emprendedores experimentan emociones muy intensas en sus decisiones conforme muestran un mayor compromiso hacia sus proyectos emprendedores.

Existen numerosos estudios que evidencian los efectos benéficos de las emociones positivas sobre los individuos, incluso emociones como la pasión, el entusiasmo y el afecto proveen impulsos importantes en el proceso de emprendimiento (Baum \& Locke, 2004; Cardon et al., 2005; Smilor, 1997). Los estudios también han probado que las emociones positivas permiten tener una mayor eficiencia en la toma de decisiones (Estrada, 1997; Isen, 2000), un mayor involucramiento en tareas (Lybomirsky et al., 2005) y comportamiento de aproximación a resultados (Baron, 2000a). Adicionalmente, las emociones positivas podrian explicar por qué algunos emprendedores son capaces de tolerar intensos niveles de estrés (Baron, 2008) y pueden tolerar una mayor presión externa.

Algunos estudios (Ardichvili et al., 2001; Baron, 2004; Baron, 2008 y Forgas, 2000) han demostrado los efectos negativos de las emociones positivas (tales como jovialidad $\mathrm{y}$ felicidad): pueden hacer que el emprendedor no evalúe todas las posibles alternativas potenciales y tome decisiones de forma prematura. Este escenario se da cuando el emprendedor deja de buscar información y toma una decisión muy rápida, animado por una idea o intuición y creyendo que no encontrará una mejor. También se ha demostrado que las emociones positivas incrementan la voluntad de asumir riesgos; el emprendedor siente un gran optimismo que genera la creencia de que se es capaz de lidiar con problemas potenciales (Weiss, 2002) y que se obtendrán resultados positivos (Busenitz \& Barney, 1997) lo cual incrementa la tendencia a tomar decisiones exitosas. Cacioppo et al. (1993) han mostrado cómo las emociones positivas resultan ser contagiosas, generando una mayor habilidad persuasiva en inversionistas, empleados y clientes. Sin embargo no se puede asumir que las emociones positivas en general sean de más ayuda para un emprendimiento que las emociones negativas.

Se ha encontrado que las emociones negativas son consecuencia del comportamiento evitativo (Krause, 2004, Lazarus et al., 1980). Algunos estudios han mostrado que las emociones negativas pueden tener una influencia positiva en el proceso de toma de decisiones por medio de una mayor concentración y un mejor procesamiento de los detalles (Schwarz et al., 1991). Sin embargo, las emociones negativas pueden hacer que los emprendedores tengan más conductas de aversión al riesgo de tal manera que sólo toman decisiones cuando se sienten completamente seguros con el fin de minimizar los riesgos y evitar los resultados negativos. Higgins (2005) y Brockner et al. (2004) llaman a este fenómeno "enfoque de prevención", el cual salvaguarda al emprendedor de involucrarse en acciones emprendedoras aunque estas pudieran ser beneficiosas. Shepherd (2003, 2004) encontró que para los emprendedores resulta ser más motivadora una emoción de miedo al fracaso que una emoción orientada al éxito.

Se ha encontrado también que las emociones influencian la percepción del mundo externo (Baron, 2008; Forgas 1995, 2000). Las personas que muestran emociones positivas tienden a percibir el mundo externo de una forma más positiva, mientras que las personas con emociones negativas, tienden a percibirlo de forma más negativa (Baron, 2008). En línea con esto, los emprendedores con emociones positivas tienden a percibir un rango más amplio de estímulos que los emprendedores con emociones negativas (Isen, 2002; Schiffman, 2005). Las emociones positivas ensancharian el estado de alerta del emprendedor (Baron, 2008), los estados de creatividad o cognición creativa (Isen, 
1999) y la flexibilidad cognitiva, como mayores conexiones y asociaciones entre ideas (Ward, 2004). Sin embargo, los individuos con emociones positivas pueden estar bajo mayores posibilidades de distraerse (Dreisbach \& Goschke, 2004).

Por último, ¿cuáles son las tendencias dentro del campo de la investigación del emprendimiento desde el punto de vista de la psicologia?

Las tendencias son las siguientes: (i) La aplicación de los principios y las dinámicas de la psicología cognitiva; (ii) La contemplación de la naturaleza ontológica de las oportunidades empresariales, (iii) Un acentuado énfasis en las dimensiones sociales del proceso. Adicionalmente, otra tendencia que es claramente evidente y parece ser no deseada es (iv) el estudio de la creciente brecha entre las definiciones teóricas y operativas de las oportunidades empresariales (Baron, 2008).

La primera tendencia tiene que ver con el abordaje del emprendimiento desde la psicología cognitiva. Indudablemente ésta ha sido la principal tendencia en los últimos ocho años de investigación.

La principal pregunta que se plantea esta orientación es acerca de cómo los ambientes en el mercado están representados en la mente de los empresarios, de tal forma que detecten y exploten las oportunidades. Más específicamente una explicación cognitiva del proceso empresarial de detección de oportunidades debe responder: (i) Cómo el esquema mental de un empresario difiere significativamente del que no tiene mente de empresario o emprendedor; (ii) Cómo los empresarios utilizan esta información de manera diferente a los que no son empresarios y (iii) Cómo éstas propiedades únicas de sus modelos mentales los llevan a la identificación de mayores oportunidades.

La segunda tendencia se desarrolla en torno a conocer la naturaleza ontológica de oportunidades empresariales, y que busca hacer distinciones entre los diferentes tipos de oportunidades para que las futuras discusiones sean productivas.

La tercera tendencia se basa en considerar un mayor énfasis en las dimensiones sociales involucradas en el emprendimiento. Los modelos mentales que representan el mercado se desarrollan a lo largo del tiempo a través de una variedad de interacciones con otros actores del mercado a través del aprendizaje, compra, venta, trabajo, entre otros. Sin embargo, mientras el concepto de modelos mentales es primordialmente un fenómeno de nivel individual, es también un fenómeno social porque su creación requiere de interacción social.

La última tendencia tiene que ver con la ampliación de las brechas en la definición de las oportunidades empresariales. Existe una gran distancia entre las definiciones teóricas y empíricas de las oportunidades empresariales. Existen tres conceptos básicos sobre las oportunidades empresariales publicados desde la época de Shane \& Venkataraman (2000) (i) Introducir al mundo nuevas materias primas, productos, servicios o procesos; (ii) Empezar un negocio; (iii) Introducir al mundo nuevas materias primas, productos, servicios o procesos a través de un nuevo negocio.

Debido a la situación actual que se está viviendo en el mundo las nuevas industrias, la tecnología, el calentamiento global, entre otros, deben surgir nuevos productos y servicios que deberán entrar a competir en el mercado, por lo que deberá haber una cantidad significativa de identificación de nuevas oportunidades.

Estas circunstancias permiten dos vias de investigación: la primera es hacer predicciones acerca de cómo las estructuras industriales se desarrollaran, la segunda nos permite analizar a través de los estudios teóricos estudiar los modelos mentales y cómo cambian y evolucionan con el paso del tiempo.

\section{Método}

Teniendo como punto de partida el propósito de este estudio de reconocer los principales procesos psicológicos involucrados en el proceso de emprendimiento empresarial, con el fin de identificar las acciones necesarias para construir una propuesta de abordaje de los contenidos del emprendimiento para los estudiantes del Politécnico Grancolombiano, el enfoque que se utilizará será de corte cualitativo ya que en palabras de Bonilla y Rodríguez (1997) "en la investigación cualitativa, se pretende captar el conocimiento, el significado y las interpretaciones que comparten los individuos sobre la realidad social que se estudia y es definida como un producto histórico, es 
decir, es validada y transformada por los mismos sujetos" (p. 52).

\subsection{Participantes}

En la investigación de corte cualitativo no se busca que los resultados sean susceptibles de generalización, el propósito de este tipo de investigación radica en explorar la comprensión que tienen los sujetos sobre la realidad y los significados que tienen alrededor de ésta. Por esta razón, para el presente estudio, la selección de la muestra se realizó por medio del muestreo intencional, que consiste en elegir criterios que se consideran importantes para tener una unidad de análisis con las mayores ventajas para conseguir los fines de la investigación. (Valles, 1997)

El criterio para seleccionar los participantes fue: ser un reconocido emprendedor con experiencia el liderar y gestionar un negocio propio durante al menos cinco años. Se realizaron entrevistas en profundidad a cinco emprendedores.

\subsection{Técnicas de recolección de información}

Se utilizó la entrevista a profundidad a expertos en el tema de emprendimiento. Se ha escogido la entrevista debido a que se tiene por objetivo recoger un conjunto de saberes privados, la construcción del sentido social de la conducta individual o del grupo de referencia. Entendemos la entrevista a profundidad desde la definición dada por Taylor y Bogdan "los reiterados encuentros, cara a cara, entre el investigador y los informantes, encuentros éstos dirigidos hacia la comprensión de las perspectivas o situaciones, tal como las expresan con sus propias palabra" (P. 78).

Especificamente se trabajará la entrevista a profundidad, porque suele cubrir solamente unos pocos temas pero en mayor profundidad y donde emergen preguntas que el investigador hace a partir de las respuestas del entrevistado, y se centran fundamentalmente en la aclaración de los detalles con la finalidad de profundizar en el tema objeto de estudio.

\subsection{Instrumentos}

Para el presente trabajo de investigación uno de los dos instrumentos de recolección de información será la entrevista a profundidad, que sigue el modelo de una conversación de igual a igual y no sólo de un intercambio formal de preguntas y respuestas.

\section{Resultados}

El proceso de trabajo de campo consistió en acercarnos a emprendedores reales con empresas exitosas y una trayectoria de más de cinco años en el mundo empresarial; la intención no era confirmar elementos trabajados en el aparato teórico sino agregar valor al conocimiento sobre el emprendimiento con la experiencia real de emprendedores verdaderos. El reto para el investigador y el semillero consistió en observar marcos mentales, percepciones, motivaciones, emociones y comportamientos de estos emprendedores y reflexionar a propósito de cómo la formación en una cátedra de emprendimiento podría formar a candidatos a ser emprendedores y prepararlos para los retos que supone un ambiente empresarial.

A propósito del tema del mundo y la vida del emprendedor, se pudieron establecer los beneficios que encuentran los entrevistados en su actividad, como una mayor libertad e independencia para escoger la forma de hacer las cosas, en este sentido el liderazgo es entendido como la oportunidad de ser creativo e innovador por la propia mano, y aunque es necesario responder a inversionistas, entre más éxito tiene la actividad emprendedora es mayor la autonomía que desarrolla el emprendedor. Es importante notar que los entrevistados están de acuerdo al decir que la confianza en sí mismos es un factor crítico a la hora de decidirse a emprender un nuevo negocio, y que esta confianza se incrementa con cada buena decisión.

El grupo investigador indagó por los errores de percepción relacionados con la excesiva confianza en sí mismo y en la sobreestimación de las oportunidades: para los entrevistados el riesgo es una actitud que conlleva una orientación hacia la oportunidad y no tanto hacia la pérdida. Uno de ellos afirmaba que de hecho habia nacido pobre y que no era mucho lo que tenía que perder pero sí mucho lo que podría 
ganar. Todos los emprendedores entrevistados tuvieron negocios o lineas de producto que fracasaron, sin embargo su forma de representarlo es como un riesgo que vale la pena tomar por los privilegios de aprendizaje y el conocimiento de los propios límites; todos lo volverian a intentar.

La toma de decisiones es un proceso cognitivo que está vinculado a las emociones, y que los entrevistados definen como una especie de instinto que les permite sentir cuando algo está por el camino equivocado y cuando una certeza significa que, aunque no existan datos, es necesario lanzarse.

Uno de ellos narró una historia que es de por sí muy interesante y merece la pena consignarse: era la historia de un emprendedor y presidente de una empresa de televisión por cable quien invirtió una suma muy elevada en un estudio que al final le aconsejó alguna decisión sobre segmentación de su servicio que era de por sí muy arriesgada. Los datos proyectaban un mejoramiento de las finanzas como producto de tomar la decisión; sin embargo, y pese al análisis de diversos expertos, decidió ignorar la abundante información y no tomó la decisión recomendada. El resultado fue que al final los resultados financieros no se dieron y esto implicó la posterior fusión con otra empresa más grande que la adquirió por un precio bajo. La influencia de las emociones y del instinto puede nublar la toma de decisiones informadas. El emprendedor tiene que aprender a gerenciar con personas con un amplio conocimiento específico y necesita herramientas con las cuales pueda valorar este conocimiento y dialogar con estos profesionales de una manera en que la organización reciba valor agregado. La formación en gestión del conocimiento resulta vital, de acuerdo a los entrevistadores, en el proceso de educación para el emprendimiento.

Los entrevistados mencionan que el emprendimiento conlleva una mayor responsabilidad en las evaluaciones personales y mayor asunción de riesgos. En otras palabras, el emprendimiento conlleva un liderazgo que hace que el emprendedor sea la cabeza visible de evaluaciones de los grupos de interés y esto implica que los riesgos asumidos tienen que justificarse ante más personas de los grupos de interés. Esto tiene una contraparte, reconocida por dos de los entrevistados; el autoconcepto del emprendedor crece conforme tiene que asumir más riesgos, tomar decisiones y asumir responsabilidades, esto puede enturbiar la percepción y la interpretación de los datos.

Una de las actividades con las que tiene que lidiar el emprendedor es la incertidumbre diaria y la aparición de constantes problemas y contingencias de demandan su criterio, desde pequeños detalles hasta grandes cambios en la industria. Esto hace que el emprendedor se enfrente a una mayor vulnerabilidad a los cambios y a cómo vivirlos; esto se puede evidenciar en una mayor presión y una mayor exposición a factores de estrés laboral y personal que requieren que el emprendedor sea entrenado en toma de decisiones bajo entornos dificiles, con poca información y con una gran probabilidad de riesgo.

La integración entre trabajo y vida familiar se hace más pronunciada en la opción por el emprendimiento; de acuerdo a los entrevistados es necesario que el aspirante a emprendedor entienda que la opción que toma requiere una preparación para un cierto tipo y estilo de vida, más que negocios se trata de un proyecto de vida. Sin embargo, los entrevistados reconocen que existe una motivación a la recompensa que se vincula al esfuerzo invertido en un proyecto, esta motivación permite que la toma de riesgos se una a la expectativa de retribución y premio por su labor. Existe una recompensa que los entrevistados admiten como interesante, y es el reconocimiento social y el incremento de estatus como fruto del esfuerzo empresarial. Algunos de los entrevistados, sin embargo, reconocen que esto en lugar de ser una recompensa se convierte en un factor más de presión, en la medida en que guardar las apariencias o la reputación es algo trascendental cuando la imagen personal está asociada a la imagen de la marca, producto o servicio ofrecido.

El principal motivador para el emprendedor es la motivación del reto, que los entrevistados reconocen en la búsqueda diaria de nuevas oportunidades en el segmento de mercado que han escogido. Esta motivación los lleva a prepararse y adquirir nuevos conocimientos. Los entrevistados en su mayoría se definen como "buscadores de conocimiento", se dejan llevar por su intuición para escoger información, asistir a eventos de formación y capacitación y superarse cada día a sí mismos. La orientación al logro puede encontrarse también en los entrevistados, quienes reconocen que constantemente se mantienen actualizados con el impulso de lograr colonizar nuevos mercados y nuevos consumidores. Algunos emprendedores 
habian tenido preparación previa y conocimientos en administración, gerencia, finanzas $\mathrm{y}$ gestión de recursos humanos $\mathrm{y}$ lograron capitalizar sus proyectos utilizando estos conocimientos. La mayoría de emprendedores entrevistados, sin embargo, reconoce que cuando iniciaron su proyecto no tenían mayores conocimientos de las áreas de conocimiento vinculadas a la gestión y la administración, sin embargo tenían la motivación para buscar capacitarse e informarse, este proceso se dio simultáneo a la implementación de su iniciativa emprendedora.

Dentro de la creación y el diseño de la organización emprendedora es necesario tener en cuenta, de acuerdo a los entrevistados, la creación y el reforzamiento de un fuerte sentido de propiedad, el refuerzo de sentimientos de libertad y autonomia, la maximización de oportunidades a través de una administración holística (esto es, que se vincule con todo el negocio y se involucre a fondo en los detalles), la tolerancia a la ambigüedad y la toma de decisión intuitiva, la responsabilidad de desarrollar una visión, la búsqueda de construcción del compromiso a largo plazo, la necesidad de construir redes de grupos de interés relevantes y el permitir los errores dentro de una cultura de aprendizaje y de gestión del conocimiento.

\section{Discusión}

¿Hacia dónde van las tendencias de educación en emprendimiento? Aunque los estudiantes necesitan desarrollar sus habilidades y comprensión para los negocios, es necesario prestar atención al desarrollo de las habilidades, atributos y comportamientos asociados al emprendimiento. Esto implica introducir nuevos módulos y cursos específicamente diseñados para desarrollar en ellos la conciencia y las características típicas de un emprendedor. De acuerdo a Ray (1997), entre los contenidos se hace necesario incluir habilidades de comunicación, -especialmente de persuasión-, habilidades creativas, habilidades de pensamiento critico, habilidades de liderazgo, habilidades de negociación, habilidades de resolución de problemas, habilidades de trabajo en equipo y construcción de redes, habilidades de manejo del tiempo y habilidades de búsqueda de información.

Estos contenidos por sí mismos no son suficientes sin la creación de ambientes de aprendizaje que cambien la manera en que los estudiantes aprenden y refuerzan, no sólo el desarrollo de habilidades, sino su capacidad de tomar propiedad y lidiar con la ambigüedad, la falta de certeza y el riesgo.

Mientras la orientación de la educación tradicional se halla centrada en el pasado, el análisis crítico, el conocimiento pasivo, la manipulación de los simbolos, la comunicación escrita, la objetividad y los conceptos, la educación para el emprendimiento debe estar basada en tomar énfasis en el futuro, la creatividad, la elaboración de puntos de vista superiores de los fenómenos, el conocimiento activo, el involucramiento emocional, la intervención en los eventos, la comunicación personal y la influencia y búsqueda de oportunidades (Gibb, 1987).

Kirby (1992), Olson y Bosserman (1984), sugieren que los individuos exhibirán comportamiento emprendedor cuando poseen una combinación de tres atributos: orientación al rol, que enfatiza la efectividad; habilidades para pensar intuitiva y racionalmente; y motivación, entendida como la fuerza orientadora detrás de la acción. Para lograr estos tres atributos es necesario adoptar una aproximación al aprendizaje que tenga los siguientes atributos:

-Primero: dar a los estudiantes propiedad sobre su propio aprendizaje, incluyendo la posibilidad de negociar con su profesor sus propios objetivos de aprendizaje, los recursos, actividades y procesos requeridos para conseguir estos objetivos $\mathrm{y}$, sobre todo, la forma en la cual se determinará si los objetivos han sido conseguidos, con el fin de estimular la motivación, reducir la dependencia y proveer experiencia de orientación de rol.

-Segundo: involucrar a los estudiantes en la solución de problemas en contextos reales y situaciones del mundo real. Orientar esta solución al trabajo en equipos, con el fin de desarrollar tanto el pensamiento intuitivo como el pensamiento racional, para reconocer la naturaleza polifacética de los problemas y soluciones y para propiciar la comunicación y la cooperación.

-Tercero: animar a los estudiantes a formular decisiones sobre la base de información inmediata, incompleta, dudable, así como información apropiada y generada por ellos mismos, esto 
con el fin de estimular la efectividad y la habilidad de decidir en ambientes inciertos.

-Cuarto: proveer a los estudiantes de modelos que estén involucrados tanto en el proceso de aprendizaje como en el de valoración, con el fin de evidenciar la orientación de rol, la habilidad y la motivación.

Ha sido demostrado por diversos autores, que los comportamientos, habilidades y atributos no son exclusivos de ciertos individuos pero pueden ser dominantes en unos individuos más que en otros. Diferentes individuos pueden tener una mezcla diferente y quizá una propensión diferente para desarrollar su capacidad. Estos comportamientos pueden ser practicados, desarrollados y aprendidos en algún grado, y ciertos ambientes; particularmente los relacionados con administrar un negocio propio, los propician de forma privilegiada (Gibb, 2000).

Se ha hecho una distinción entre comportamiento emprendedor efectivo (definido como realizar metas personales, organizacionales y sociales), y comportamiento emprendedor no efectivo (definido como basado en decisiones erráticas, con desperdicio de recursos y consecución nula o parcial de metas).

Definir el emprendimiento en términos de comportamientos, habilidades y atributos necesarios para responder a problemas y oportunidades en un ambiente social, implica educar para contingencias y ciertas fortalezas individuales relacionadas con rasgos de comportamiento y diseño de organizaciones y planes de negocio. La definición de emprendimiento ha dejado campo a un modelo de emprendimiento que, siguiendo la metáfora del cuerpo humano, toma en cuenta los siguientes elementos:

Un corazón de valores que se constituye en el objetivo más importante de un programa de educación en emprendimiento, donde se hace acento en la empatía y la adopción de valores emprendedores. Estos valores están asociados a la forma en que se hacen las cosas, en que se organizan, se sienten, se comunican, se comprenden y se piensan (Gibb, 2002a). Una lista de valores emprendedores asociados con "formas de hacer las cosas" y "formas de ser" son:
El instinto estratégico, el cual ha sido visto a lo largo de sucesivas investigaciones que han mostrado que el pensamiento estratégico y la orientación estratégica son la clave de los atributos emprendedores (Atherton, 1997; Gibb $\&$ Scott, 1985). El pensamiento estratégico involucra una mezcla dinámica de valoración del futuro de la organización y cómo puede ser llevada a ese horizonte futuro; constante análisis de "qué pasaría si" aplicado a eventos y su impacto potencial en los negocios y la estrategia; ver el negocio siempre a partir de los ojos de los grupos de interés claves; buscar $\mathrm{y}$ perseguir las nuevas tendencias de gustos y elecciones de los consumidores; constante monitoreo del entorno de negocios; conocimiento claro del estado del negocio, sus debilidades y fortalezas.

El crecimiento de atributos emprendedores requiere pedagogias y metodologías diferentes. Ha habido una larga historia de simulaciones y juegos de rol diseñados para este propósito (McClelland y Winter, 1969; Spencer, 1983). El drama, por ejemplo, puede ser usado para calificar la empatía con grupos de interés, para valorar las estrategias de búsqueda de información y para desarrollar habilidades comunicativas y persuasivas.

La inteligencia emocional subraya la importancia del manejo de los sentimientos y afectos (Dulewicz, 2000, Goleman, 1996). En el centro de una gerencia exitosa de redes, socios y colaboradores se ubica el manejo emocional, la auto conciencia emocional, el manejo y armonización de las emociones con el proceso emprendedor, de toma de decisiones y asunción de riesgos. Así mismo es importante desarrollar la capacidad de leer las emociones de otros y comprender cómo las emociones impactan las comunicaciones y las acciones, esto con el fin de aplicar estos conocimientos en el proceso de gerencia y administración. Es de vital importancia conocer cómo la comunicación se encuentra afectada por los sentimientos que se hallan subyacentes a los valores identificados arriba y cómo estos sentimientos impactan la forma en que percibimos las cosas.

Es importante que el educador trabaje en la visión de proyecto de vida dentro del mundo empresarial. La labor de la universidad sería ayudar a los estudiantes a entender el mundo y la vida de una persona emprendedora dentro de una organización emprendedora. Una forma de crear esta visión es preguntar a los estudiantes qué cosas cambiarían al momento 
de convertirse en emprendedores. El programa de educación en emprendimiento tendría como reto buscar la manera de construir capacidades para ser excelente en ese cambio y disfrutarlo. Otra estrategia interesante es la de realizar junto con los estudiantes entrevistas a un amplio rango de individuos en su comunidad (trabajadores dependientes, informales, emprendedores, estatales, voluntarios) con el fin de explorar las motivaciones y grados de incertidumbre de cada categoría de trabajo; el objetivo es prepararlos para la transición al mundo del trabajo y al mundo del emprendimiento.

Otra herramienta importante para desarrollar, es la capacidad de usar conocimiento basado en la experiencia y la experimentación. Los escritos de Lave y Wenger (1991) sobre la importancia del aprendizaje situado subrayan esto. En términos pedagógicos el reto consiste en maximizar la oportunidad para el uso y la práctica del conocimiento adquirido, de tal forma que sea posible simular el mundo del emprendedor por medio del aprendizaje, del proceso de investigar, hacer, implantar, aprender y cuestionar el conocimiento.

El desarrollo cognitivo es el enfoque central de varias aproximaciones orientadas a la recepción del conocimiento, reconocimiento, juicio y recuerdo. De acuerdo a Ruohotie \& Karanen (2000), el énfasis de la educación para el emprendimiento debe estar ubicado en los aspectos afectivos y motivacionales del aprendizaje. El desarrollo afectivo tiene que ver con la respuesta individual, los gustos, disgustos, sentimientos, emociones y estados de ánimo. El desarrollo motivacional tiene que ver con el impulso de dar sentido a la información. Cada uno de estos elementos resulta ser importante en el proceso de aprendizaje emprendedor y hasta ahora ha parecido no tener mucha relevancia dentro del diseño curricular de los programas de negocios.

El reto de los emprendedores independientes es gerenciar el negocio como un todo integrado. E1 reto para los educadores es desarrollar la capacidad de anticipar contingencias y problemas, buscar conocimiento constantemente para prever tendencias y adaptarse a los cambios. Este énfasis en el problema no significa que el análisis conceptual se sacrifique, sino que se hace énfasis en el proceso de exploración de problemas que permita encontrar en ellas oportunidades.
Dentro del proceso de educación y formación para el emprendimiento no se puede pasar por alto la formación en la capacidad de "aprender a aprender" a partir de las relaciones con los grupos de interés del entorno. Crear la capacidad para el aprendizaje a partir de relaciones es el secreto para construir relaciones honestas. La clave de este enfoque es identificar lo que los emprendedores necesitan saber de cada grupo de interés en cada fase del desarrollo de su organización.

Dentro de este proceso, la labor del educador es ayudar al estudiante a identificar las oportunidades en el contexto del individuo que pueden propiciar un comportamiento emprendedor, y como corolario de esto, el objetivo es ayudar a ese estudiante a desarrollar la habilidad de diseñar organizaciones en diferentes contextos y necesidades. Teniendo en cuenta la definición que da Gibb (1999) sobre organizaciones emprendedoras, éstas son aquellas que maximizan el potencial para los individuos que se encuentran dentro de ella y que propician el comportamiento emprendedor generando, en virtud de éste, satisfacción personal y desarrollo de las potencialidades de la organización.

La identificación de oportunidades y la implementación de las mismas es uno de los grandes retos de la formación para el emprendimiento. El proceso por el cual se identifican necesidades y se combinan dentro de conceptos de productos y servicios es fundamental en el proceso de comportamiento emprendedor y se puede encapsular dentro de la formación en gerencia de proyectos. La clave del éxito es que este proceso no sea demasiado formal o burocratizado, sino que deje campo a la creatividad, la experimentación, el descubrimiento y el aprendizaje a través de los errores.

La formación ética en consumo sostenible, en responsabilidad social y en creatividad e innovación, son críticas dentro del proceso de emprendimiento. Gibb (1999) señala como una de las fallas más notables en el comportamiento emprendedor el juego sucio, la trampa y la ventaja que algunos emprendedores aplican en su particular forma de hacer negocios y diseños organizacionales que defraudan a los grupos de interés, a consumidores, inversionistas y empleados. La inteligencia moral resulta critica para que las motivaciones intrínsecas del proceso de emprendimiento no terminen por volver al individuo en un ser egoísta, utilitarista e inescrupuloso que no le importan los medios con tal de lograr sus fines. 
Por último, una clave imperativa en el diseño de un modelo de formación en emprendimiento es identificar las fuentes de incertidumbre y complejidad que crean la necesidad de una sociedad orientada hacia el emprendimiento. Ha sido mostrado por autores como Gibb (1999) que una aproximación útil consiste en explorar el impacto de la globalización en la sociedad como un todo, desde el diseño de organizaciones hasta la vida de los trabajadores, consumidores y miembros de familias. E1 valor de este énfasis reside en que, en general, la globalización parece ser el disparador de politicas enfocadas en el emprendimiento. El reto es ver este tema más allá de la orientación de mercadeo a partir de un flujo de certezas e incertidumbre que permean la actividad de organizaciones y que van más allá de las simples presiones del mercado.

Un modelo de formación en el emprendimiento implica considerar una mayor integración del conocimiento (dentro de facultades y departamentos, entre varias disciplinas de las ciencias sociales, entre campos de artes y ciencias y entre conocimiento tácito y explícito) e implica también un mayor esfuerzo de enseñanza interdisciplinaria, pues como lo ha mostrado este trabajo, la labor de pedagogía debe estar dirigida por administradores, psicólogos, antropólogos, artistas, sociólogos y un amplio espectro profesional. Este modelo requiere abrir el espacio al desarrollo del conocimiento basado en la experiencia, demanda considerar una innovación en los procesos de evaluación y acreditación y por último requiere cambios en las bases filosóficas del aprendizaje, particularmente en el campo de la administración (Ghostal, 2005; Mintzberg, 2004). 


\section{Referencias}

1. Atherton, A. (1997), "Strategy and strategic planning. Business plans and planning: literature review". High Growth Companies Project, Durham University Business School.

2. Bagozzi, R. (1992). The selfregulation of attitudes, intentions, and behavior. Social Psychology Quarterly 55: 178-204.

3. Bagozzi, R, Dholakia U. (1999). Goal Setting and Goal Striving in Consumer Behavior. Journal of Marketing, 63: 19-32.

4. Bagozzi, R., Dholakia U., Basuroy S. (2003). How effortful decisions get enacted: The motivating role of decision processes, desires, and anticipated emotions. Journal of Behavioral Decision Making 16: 273-295.

5. Bagozzi, R., Warshaw P. (1992). An examination of the etiology of the attitude-behavior relation for goal-directed behaviors. Multivariate Behavioral Research 27: 601-634.

6. Bandura, A. (1986). Social Foundations of Thought and Action: A Social Cognitive Theory. Prentice Hall, Englewood Cliffs.

7. Bandura, A. (1989). Regulation of Cognitive Process Through Perceived Self-Efficacy. Developmental Psychology 25: 729-735

8. Baron, R. A. (1998). Cognitive mechanisms in entrepreneurship: why and when entrepreneurs think differently than other people. Journal of Business Venturing 13:275-294

9. Baron, R. A. (2000a). Counterfactual thinking and venture formation: the potential effects of thinking about "what might have been". Journal of Business Venturing 15:79-91.
10. Baron, R. A. (2000b). Psychological perspectives on entrepreneurship: cognitive and social factors in entrepreneurs' success. Current Directions in Psychological Science 9:15-18.

11. Baron, R. A. (2008). The role of affect in the entrepreneurial process. Academy of Management Review 33:328-340.

12. Barringer, B. R. \& Ireland, R. D. (2006). Entrepreneurship: Successfully launching new ventures. Pearson, Upper Saddle River, NY.

13. Baum, J. R., Locke, E. A. (2004). The relationship of entrepreneurial traits, skill, and motivation to subsequent venture growth. Journal of Applied Psychology 89:587-598.

14. Bhave, M. P. (1994). A process model of entrepreneurial venture creation. Journal of Business Venturing 9: 223-242.

15. Bonilla, E. \& Rodríguez, P. (1997). Más allá del dilema de los métodos: La investigación en ciencias Sociales. Bogotá: Ediciones Uniandes-Editorial Norma.

16. Boyd, N. G. \& Vozikis, G. S. (1994). The impact of self-efficacy on the development of entrepreneurial intentions and actions. Entrepreneurial Theory and Practice 18: 63-77.

17. Brockhaus, R. H. Sr, Horwitz P. S. (1986). The psychology of the entrepreneur. In Sexton DL, Smilor RW (eds), The Art and Science of Entrepreneurship. Cambridge, Ballinger, pp. 25-48.

18. Brockner, J. Higgins ET, Low M. B. (2004). Regulatory focus theory and the entrepreneurial process. Journal of Business Venturing 19:203-221.

19. Busenitz, L. W. \& Barney, J. B. (1997). Differences between entrepreneurs and managers in large organizations: Biases and heuristics in 
strategic decision making. Journal of Business Venturing 12: 9-30.

20. Cacioppo, J. T., Klein, D. J., Berntson, G. G., Hatfield, E. (1993). The psychophysiology of emotions. In: Lewis M, Haviland JM (eds) Handbook of emotions. Guilford Press, New York.

21. Cardon, M. S., Zietsma, C., Saparito, P., Matherne, B. P. \& Davis, C. (2005). A tale of passion: new insights into entrepreneurship from a parenthood metaphor. Journal of Business Venturing 20:23-45.

22. Carsrud, A. L \& Johnson, R. W. (1989). Entrepreneurship: A social psychological perspective. Entrepreneurship and Regional Development 1(1): 21-32.

23. Carsrud, A. L., Olm, K. W., Thomas J. B. (1989). Predicting entrepreneurial success; effects of multidimensional achievement motivation, levels of ownership, and cooperative relationships. Entrepreneurship and Regional Development, 1: 237-244.

24. Carter, N. M., Gartner, W. B., Shaver K. G. (2004). Career reasons. In: Gartner WB, Shaver KG, Carter NM, Reynolds PD (eds) Handbook of Entrepreneurial Dynamics: The Process of Business Creation. Sage, Thousand Oaks, CA, pp. 142-152.

25. Choi, Y. R., Levesque M., Shepherd, D. A. (2008). When should entrepreneurs expedite or delay opportunity exploitation? Journal of Business Venturing 23 : 333-355.

26. Cohen, A. R., Zimbardo, P. G. (1969). Dissonance and the need to avoid failure. In: Zimbardo PG (ed). The Cognitive Control of Motivation: The Consequences of Choice and Dissonance. Glenview, IL: Scott Foresman and Company.

27. Deci, E. L. (1975). Intrinsic Motivation. New York: Plenum Press.
28. Deutsch, M., Krauss, R. M. (1965). Theories in Social Psychology. New York: Basic Books.

29. Dreisbach, G. \& Goschke T. (2004). How positive affect modulates cognitive control: reduced perseveration at the cost of increased distractibility. Journal of Experimental Psychology: Learning, Memory and Cognition 30:343-353.

30. Dulewicz, V. (2000), 'Emotional intelligence: the key to successful corporate leadership', Journal of General Management, 25, 1-15.

31. Dunne, T., Roberts, M., Samuelson, L. (1988). Patterns of firm entry and exit in U.S. manufacturing industries. Rand Journal of Economics 19: 495-515.

32. Elfving, J. (2008). Contextualizing Entrepreneurial Intentions: A Multiple Case Study on Entrepreneurial Cognitions and Perceptions. Turku, Finland: Åbo Akademi förlag.

33. Entrepreneurship, Innovation, and Change, 1 (2), pp. 165-6.

34. Estrada, C. A., Isen A. M., Young, M. J. (1997). Positive affect facilitates integration of information and decreases anchoring in reasoning among physicians. Organizational Behavior and Human Decision Processes 72:117-135.

35. Festinger, L. (1957). A Theory of Cognitive Dissonance. Evanston, IL: Row, Peterson. Fiet JO (1996) The informational basis of entrepreneurial discovery. Small Business Economics 8: 419-430.

36. Fisher, I. (1930) The Theory of Interest. New York: MacMillan.

37. Forgas, J. P. (1995). Mood and judgment: the affect infusion model (AIM). Psychological Bulletin 117:39-66. 
38. Forgas, J. P. (2000). Feeling and thinking: affective influences on social cognition. Cambridge University Press, New York

39. Freud, S. (1924). Introducción general al Psicoanálisis. Madrid: Amorrortu Editores.

40. Gibb, A. A. (1999). "Can we build 'effective' entrepreneurship through management development?", Journal of General Management, 24 (4), 1-22.

41. Gibb, A. A. (2000). "Corporate restructuring and entrepreneurship: what can large organisations learn from small?", Enterprise and Innovation Management Studies, 1 (1), May, 19-37.

42. Gibb, A. A. (2002a), "In pursuit of a new entrepreneurship paradigm for learning: creative destruction, new values, new ways of doing things and new combinations of knowledge", International Journal of Management Reviews, 4 (3), 233-69.

43. Gibb, A. A. \& Scott, M. (1985). "Strategic awareness, personal commitment and the process of planning in the small business", Journal of Management Studies, 22 (6), November, 597-632.

44. Gimeno, J., Folta, J., Cooper, A. Woo, C. (1997). Survival of the fittest? Entrepreneurial human capital and the persistence of underperforming firms. Administrative Science Quarterly, 42: 750-783

45. Goleman, D. (1996). La inteligencia emocional. Buenos Aires: Javier Vergara.

46. Gollwitzer, P., Brandstätter, V. (1997). Implementation intentions and effective goal pursuit. Journal of Personality and Social Psychology 73: 186-199.

47. Goss, D. (2005). Entrepreneurship and 'the social': towards a deference-emotion theory. Human Relations 58:617-636.

48. Goss, D. (2007). Enterprise ritual: a theory of entrepreneurial emotion and exchange. British Journal of Management 19:120-137, OnlineEarly Articles.

49. Higgins, E. T. (2005). Value from regulatory fit. Current Directions in Psychological Science 14: 209-213.

50. Homans, G. E. (1961). Social Behavior: Its Elementary Forms. Harcourt: Brace\&World.

51. Isen, A. M. (1999). Positive affect. In: Dalgleish T, Power MJ (eds) Handbook of cognition and emotions. John Wiley \& Sons, Chichester.

52. Isen, A. M. (2000). Positive affect and decision making. In: Lewis M, Haviland Jones JM (eds) Handbook of emotions. Guilford Press, New York.

53. Isen, A. M. (2002). Missing in action in the AIM: positive affect's facilitation of cognitive flexibility, innovation, and problem solving. Psychological Inquiry 13: 57-65.

54. Kirby, D. A. (1992). "Developing graduate entrepreneurs: the UK Graduate Enterprise Programme", Entrepreneurship, Innovation and Change, 1:2, pp165-175.

55. Entrepreneurship, Innovation, and Change, 1 (2), pp. 165-6.Kirzner I. M. (1997). Entrepreneurial discovery and the competitive market process: An Austrian.

56. Koch, S. (1956). Behavior as "intrinsically" regulated:Work notes toward a pre-theory of phenomena called "motivational". Nebraska symposium on motivation 4: 42-87.

57. Krueger, N. F. (1993). The impact of prior entrepreneurial exposure on perceptions of new venture feasibility and 
desirability. Entrepreneurship Theory and Practice 18: 5-21.

58. Krueger, N. F., Brazeal D (1994) Entrepreneurial potential and potential entrepreneurs. Entrepreneurship Theory and Practice 18: 91-104.

59. Krueger, N. F., Carsrud A. L. (1993). Entrepreneurial intentions: Applying the theory of planned behavior. Entrepreneurship and Regional Development 5: 315-330.

60. Krueger, N. F. \& Dickson P. (1994). How believing in oneself increases risk taking: Perceived self-efficacy and opportunity recognition. Decision Sciences 25: 285-400.

61. Krueger, N. F., Reilly M., Carsrud A. L. (2000). Competing models of entrepreneurial intentions. Journal of Business Venturing 15: 411-532.

62. Lave, J. \& Wenger, E. (1991). Situated Learning: Legitimate Peripheral Participation, Cambridge: Cambridge University Press.

63. Lazarus, R. S., Kanner, A. D. \& Folkman, S. (1980). Emotions: A cognitive-phenomenological analysis. In: Plutchik R, Kellerman H (eds) Emotion. Theory, Research, and Experience. Academic Press, New York.

64. Lent, R., Brown, \& S., Hacket, G. (1994). Toward a unifying theory of career and academic interests, choice, and performance. Journal of Vocational Behavior 45: 79-122.

65. Locke, E. A. \& Latham, G. P. (2002). Building a practically useful theory of goal setting and task motivation. American Psychologist 57: 705-717.

66. Locke, E. A. \& Latham, G. P. (2002). Building a practically useful theory of goal setting and task motivation. American Psychologist 57: 705-717.
67. Locke, E. A., Latham, G. P. (2004). What should we do about motivation theory? Six recommendations for the twenty-first century. Academy of Management Review 29: 388.

68. McClelland, D. C., Atkinson, J. W., Clark, R. A. \& Lowell, E. L. (1953). The Achievement Motive. New York: Appleton-Century-Crofts.

69. McClelland, D. C. \& Winter, DG (1969) Motivating Economic Achievement. New York: Free Press.

70. McClelland, D.C. \& Winter, D. G. (1969). Motivating Economic Achievement, New York: Free Press.

71. McMullen, J. \& Shepherd, D. A. (2006). Entrepreneurial action and the role of uncertainty in the theory of the entrepreneur. Academy of Management Review 31: 132-152.

72. Mintzberg, H. (2004). Managers not MBAs, London: Prentice Hall.

73. Morris, M., Schindehutte M. \& Allen, J. (2006). Is the business model a useful strategic concept? Conceptual, theoretical, and empirical insights. Journal of Small Business Strategy 17: 27-50.

74. Murray, H. A. (1938). Explorations in personality. New York: Oxford University Press.

75. Nuttin, J. (1984). Motivation, Planning, and Action. Leuven: Leuven University Press and Lawrence Erlbaum Associates.

76. Olson, P. \& Bosserman, D. (1984). Attributes of the entrepreneurial type. Business Horizons May-June, pp. 53-6.

77. Palich, L. E. \& Bagby, D. R. (1995). Using cognitive theory to explain entrepreneurial risk-taking: challenging conventional wisdom. Journal of Business Venturing 10:425-438. 
78. Perwin, L. (2003). The Science of Personality. Oxford: Oxford University Press.

79. Ray, D.M. (1997). 'Teaching entrepreneurship in Asia: impact of a pedagogical innovation', Entrepreneurship, Innovation and Change, 6 (3), pp. 193-227.

80. Reynolds, P. D., Bygrave W. D., Autio E., Cox L., Hay M. (2002). Global Entrepreneurship Monitor: 2002, Executive Report. Kansas City, MO: Kauffman Center for Entrepreneurial Leadership.

81. Ruohotie, P. and Karanen, N. (2000). Building Conative Constructs into Entrepreneurship Education, Tampere, Finland: University of Tampere.

82. Russo, J. E., Shoemaker PJH (1998). Decision Traps. Doubleday, New York.

83. Schiffman, H. R. (2005). Sensation and perception: an integrated approach. John Wiley \& Sons, New York.

84. Schwarz, N., Bless, H., Bohner, G. (1991). Response scales as frames of reference: the impact of frequency range on diagnostic judgments. Applied Cognitive Psychology 5:37-49.

85. Shane, S. \& Venkataraman S. (2000). The promise of entrepreneurship as a field of research. Academy of Management Review 25:217-226.

86. Shepherd, D. A. (2003). Learning from business failure: propositions of grief recovery for the selfemployed. Academy of Management Review 28:318-328.

87. Shepherd, D. A. (2004). Educating entrepreneurship students about emotion and learning from failure. Academy of Management Learning and Education 3:274-287.
88. Shepherd, D. A., Douglas E. J., Shanley M. (2000). New venture survival: Ignorance, external shocks, and risk reduction strategies. Journal of Business Venturing 15: 393-410.

89. Simon, M., Houghton, S. M., Aquino, K. (2000). Cognitive biases, risk perception and venture formation: How individuals decide to start companies. Journal of Business Venturing 15: 115-134.

90. Sitkin, S. B. \& Pablo, A. L. (1992). Reconceptualizing the determinants of risky behavior. Academy of Management Review 17: 9-38.

91. Spencer, L. M. (1983). 'Soft skill competencies', Scottish Council for Research and Education, Edinburgh: Lindsay Company.

92. Spencer, J. (2005) Stock trading favors the fearless, study suggests. Wall Street Journal August 22:C1/C6.

93. Steel, P. \& König, C. J. (2006). Integrating theories of motivation. Academy of Management Review 31(4): 880-913.

94. Taylor, S. Y R. Bodgan (1990). Introducción a los Métodos Cualitativos de investigación. Buenos Aires: Paidós.

95. Valles, M. (1997). Técnicas cualitativas de investigación social. Reflexión metodológica y práctica profesional. Madrid: Síntesis.

96. Weiss, H. M. (2002). Deconstructing job satisfaction: separating evaluations, beliefs and affective experiences. Human Resource Management Review 12:173-194.

97. Wilson, E. O. (1998). Consilience: The Unity of Knowledge. New York: Alfred A. Knopf. 


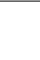

